\title{
Entanglement spectrum and entanglement thermodynamics of quantum Hall bilayers at $v=1$
}

\author{
John Schliemann \\ Institute for Theoretical Physics, University of Regensburg, D-93040 Regensburg, Germany
}

(Received 26 January 2011; published 16 March 2011)

\begin{abstract}
We study the entanglement spectra of bilayer quantum Hall systems at total filling factor $v=1$. In the interlayer-coherent phase at layer separations smaller than a critical value, the entanglement spectra show a striking similarity to the energy spectra of the corresponding monolayer systems around half-filling. The transition to the incoherent phase can be followed in terms of low-lying entanglement levels. Finally, we describe the connection between those two types of spectra in terms of an effective temperature leading to relations for the entanglement entropy which are in full analogy to canonical thermodynamics.
\end{abstract}

DOI: 10.1103/PhysRevB.83.115322

PACS number(s): 73.43.-f

\section{INTRODUCTION}

Experimental studies of quantum Hall bilayers at total filling factor $v=1$ have revealed a series of intriguing phenomena. ${ }^{1-6}$ From a theoretical point of view, such strongly interacting systems pose intricate and subtle problems of many-body physics, and many unexpected observations are still lacking a fundamental understanding. ${ }^{7}$ Here the exact numerical treatment of small systems has provided important insights and guidance of theoretical intuition.$^{8-15}$ Moreover, in the last decade many-body physics has been enriched by the concept of entanglement, whose extensive and systematic study originated in the field of quantum information theory. ${ }^{16}$ In particular, the notion of the entanglement spectrum has led to novel insights in the physics of quantum Hall monolayers at fractional filling factors, ${ }^{17-20}$ spin systems of one ${ }^{21-25}$ and two $^{26}$ spatial dimensions, quantum hall insulators, ${ }^{27,28}$ and also rotating Bose-Einstein condensates. ${ }^{29}$ The entanglement spectrum of a bipartite system is defined in terms of the Schmidt decomposition of its ground state $|\psi\rangle$,

$$
|\psi\rangle=\sum_{n} e^{-\xi_{n} / 2}\left|\psi_{n}^{A}\right\rangle \otimes\left|\psi_{n}^{B}\right\rangle,
$$

where the states $\left|\psi_{n}^{A}\right\rangle\left(\left|\psi_{n}^{B}\right\rangle\right)$ form an orthonormal basis of the subsystem $A(B)$, and the non-negative quantities $\xi_{n}$ are the dimensionless levels of the entanglement spectrum. In the present paper we study the entanglement spectrum of quantum Hall bilayers at total filling factor $v=1$, where the subsystems are naturally defined by the two layers. Our investigations are based on exact numerical diagonalizations in the spherical geometry. ${ }^{30}$

\section{RESULTS AND DISCUSSION}

Depending on the layer separation and the amplitude for particle tunneling between the layers, quantum Hall bilayers exhibit a quantum phase transition between a phase showing spontaneous interlayer coherence and quantized Hall conductance, and a phase where such effects are absent with the two layers being merely uncorrelated. The corresponding phase diagram was first mapped out experimentally by Murphy et $a l .{ }^{1}$ and later reproduced theoretically via numerical diagonalizations. ${ }^{9}$ The latter study relied on a careful analysis of fluctuations of the pseudospin describing the layer degree of freedom. Moreover, in the case of a very small tunneling amplitude, the phase transition is also signaled in experiment by the generation of a pronounced peak in the tunneling conductance at zero-bias voltage. ${ }^{2}$ Here the critical value of the layer separation of $d=1.83 \ell$ ( $\ell$ : magnetic length) was nicely reproduced by numerical calculations taking into account the finite width of the two wells. ${ }^{9}{ }^{93,31}$ In the following, however, we shall for simplicity concentrate on the case of zero well width where the transition is found numerically at $d=1.3 \ell$, and we will focus on the situation of vanishing tunneling amplitude. Moreover, we will assume the physical electron spin (as opposed to its pseudospin) to be fully polarized by the perpendicular magnetic field such that it does not play a role in the present study.

In the limit of zero layer separation $d=0$ both layers merge to a fully filled monolayer whose Coulomb ground state is given by the well-known Slater determinant of the Vandermonde type,

$$
|\psi\rangle \propto\left(\prod_{i<j}\left(z_{i}-z_{j}\right)\right) \exp \left(-\frac{1}{4 \ell^{2}} \sum_{i}\left|z_{i}\right|^{2}\right)\left|T^{z}\right\rangle,
$$

where the $z_{i}$ are the complex electron coordinates in the planar geometry. An analogous wave function exists in the spherical model of $N$ electrons where the sphere is penetrated by $N-1$ flux quanta. ${ }^{30}$ Here the $z$ component of the pseudospin can take values of $T^{z}=-N / 2, \ldots, N / 2$ meaning that, say, the top/bottom layer contains $N / 2 \pm T^{z}$ electrons. The case $T^{z}=0$ corresponds to Halperin's 111 wave function. ${ }^{32}$ Tracing out one of the layers means summing over $N / 2 \pm T^{z}$ electron coordinates in the pure state $\rho=$ $|\psi\rangle\langle\psi|$ with $|\psi\rangle$ being a single Slater determinant such that the resulting $N / 2$-body reduced-density matrix is proportional to the unit matrix with all eigenvalues being equal to $1 /\left(\begin{array}{c}N \\ N / 2 \pm T^{z}\end{array}\right)$. Thus, at $d=0$ the entanglement spectrum shrinks to a single value.

Let us now turn to the case of finite $d>0$. The Coulomb ground state of the bilayer system in spherical geometry has vanishing total angular momentum $L=0$. Therefore, the reduced density matrix is also rotationally invariant, and its eigenvalues occur in multiplets of the angular momentum. Figure 1 presents entanglement spectra obtained from balanced bilayers $\left(T^{z}=0\right)$ at small but finite-layer separation $d=0.1 \ell$ for various system sizes $N$ and compares them with the energy spectra of half-filled monolayers containing $N_{m}=N / 2$ particles. As seen from the figure, both types of 


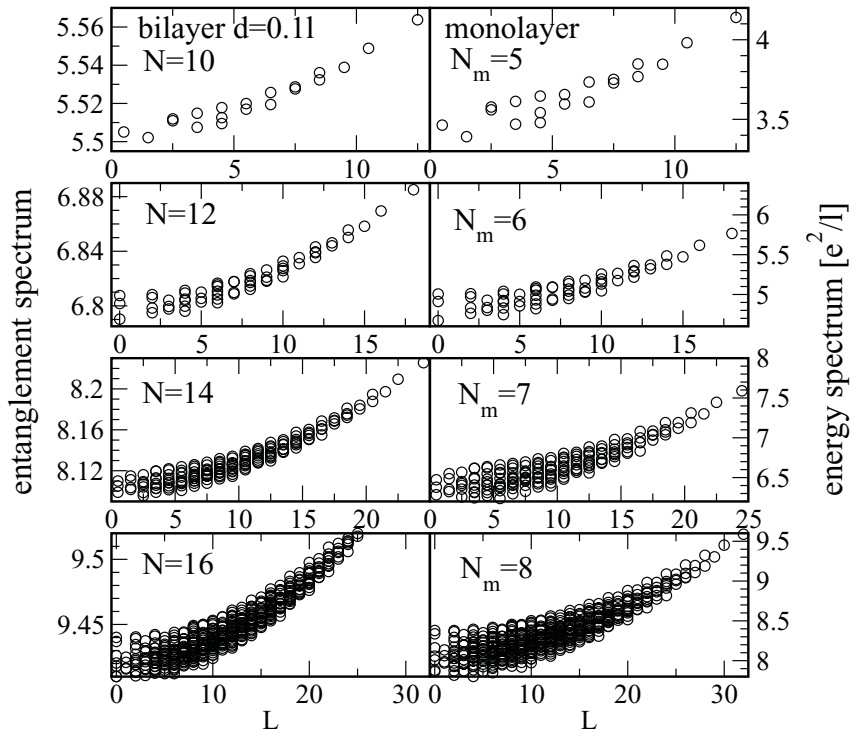

FIG. 1. Left: Entanglement spectra obtained from balanced bilayers at layer separation $d=0.1 \ell$ for various system sizes $N$. Right: Energy spectra of half-filled monolayers with $N / 2$ electrons.

spectra show a striking similarity which is, on the total scale of the spectra, particularly evident at smaller system sizes. This observation also continues to larger layer separations as shown in Fig. 2 for the cases $d=0.7 \ell$ and $d=1.3 \ell$ : With increasing layer separation the entanglement spectra widen up (while shrinking to a single level for $d \rightarrow 0$ ), but remain similar in shape to the monolayer energy spectra. At layer separations exceeding the critical value $d=1.3 \ell$ a qualitatively different behavior sets in: The global shape of the entanglement spectrum remains reasonably close to the monolayer energy spectrum, but differences are developed in details of the data. This can be seen most clearly by following the lowest entanglement level $\xi_{0}$ and the three following

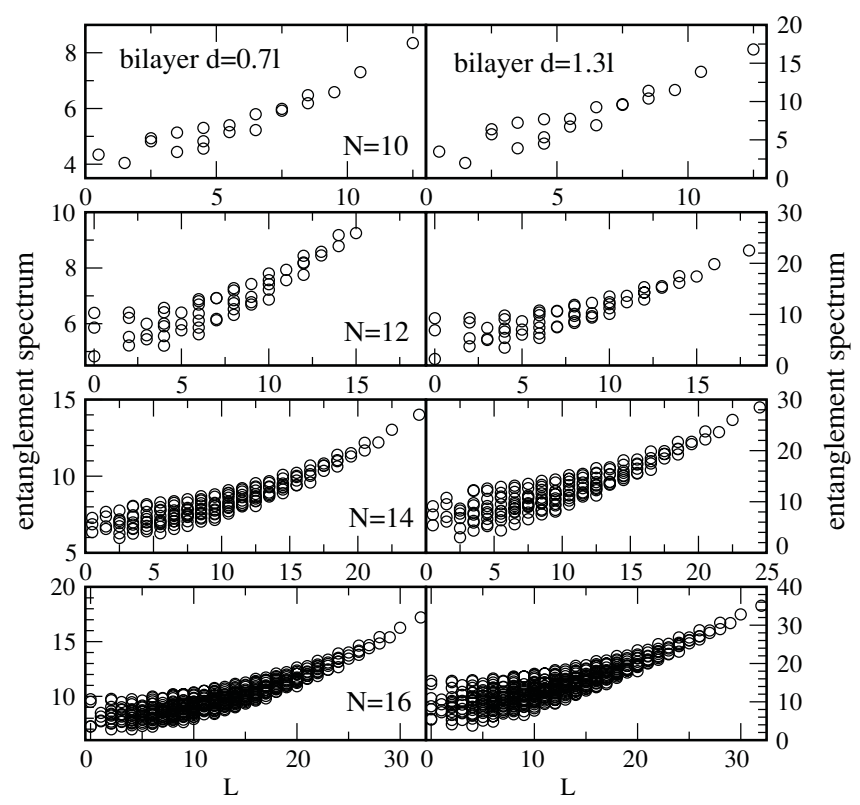

FIG. 2. Entanglement spectra of balanced bilayers at layer separation $d=0.7 \ell$ (left) and $d=1.3 \ell$ (right).
TABLE I. Angular momentum $L_{n}$ of the low-lying entanglement levels $\xi_{n}$ at various system sizes $N$ of the underlying bilayer system.

\begin{tabular}{ccccc}
\hline \hline$N$ & $L_{0}$ & $L_{1}$ & $L_{2}$ & $L_{3}$ \\
\hline 10 & $3 / 2$ & $1 / 2$ & $7 / 2$ & $9 / 2$ \\
12 & 0 & 4 & 2 & 3 \\
14 & $5 / 2$ & $11 / 2$ & $7 / 2$ & $5 / 2$ \\
16 & 4 & 2 & 6 & 0 \\
\hline \hline
\end{tabular}

levels $\xi_{n}, n \in\{1,2,3\}$, as a function of the layer separation. ${ }^{33}$ Those entanglement levels (along with their counterparts in the monolayer energy spectra) occur at different values of the angular momentum $L_{n}$ which depend on system size and are summarized in Table I.

Figure 3 shows these low-lying entanglement levels as a function of the layer separation. While at $d \lesssim 1.3 \ell$ all levels decrease with increasing layer separation, the properties of the spectrum change qualitatively at $d \approx 1.3 \ell$ : Here the excited levels start to increase with the layer separation while the lowest level approaches $\xi_{0} \approx \ln \left(2 L_{0}+1\right)$. Indeed, at large layer separations the reduced-density matrix of a single layer is dominated by a multiplet with total angular momentum $L_{0}$ with all other eigenvalues being exponentially close to zero. In this sense, the ground state of the bilayer system carrying angular-momentum quantum numbers $L=L^{z}=0$ can at large layer separation be viewed to be composed of two monolayers with $L=L_{0}$ according to a standard Clebsch-Gordan decomposition, ${ }^{34,35}$

$$
|0,0\rangle \approx \sum_{m=-L_{0}}^{L_{0}} \frac{(-1)^{L_{0}-m}}{\sqrt{2 L_{0}+1}}\left|L_{0}, m\right\rangle\left|L_{0},-m\right\rangle,
$$

leading to a reduced density matrix of the form

$$
\rho_{\text {red }} \approx \sum_{m=-L_{0}}^{L_{0}} \frac{1}{2 L_{0}+1}\left|L_{0}, m\right\rangle\left\langle L_{0}, m\right| .
$$

Thus, the entanglement entropy $S=\left\langle-\ln \rho_{\text {red }}\right\rangle,\langle\cdot\rangle:=$ $\operatorname{tr}\left(\cdot \rho_{\text {red }}\right)$, interpolates, similarly as the lowest entanglement

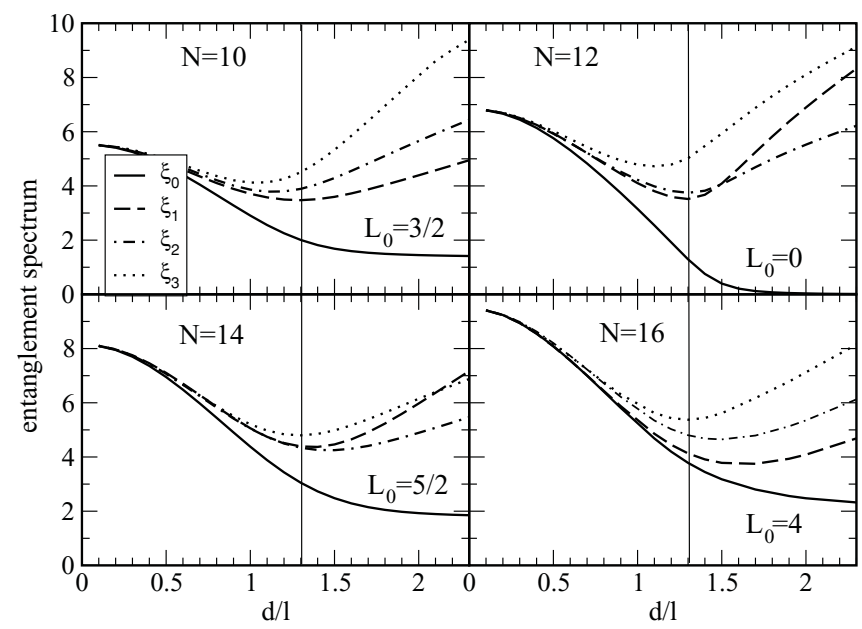

FIG. 3. The four lowest entanglement levels as a function of the layer separation for different system sizes. At the phase boundary at $d \approx 1.3 \ell$ the entanglement spectrum qualitatively changes. 

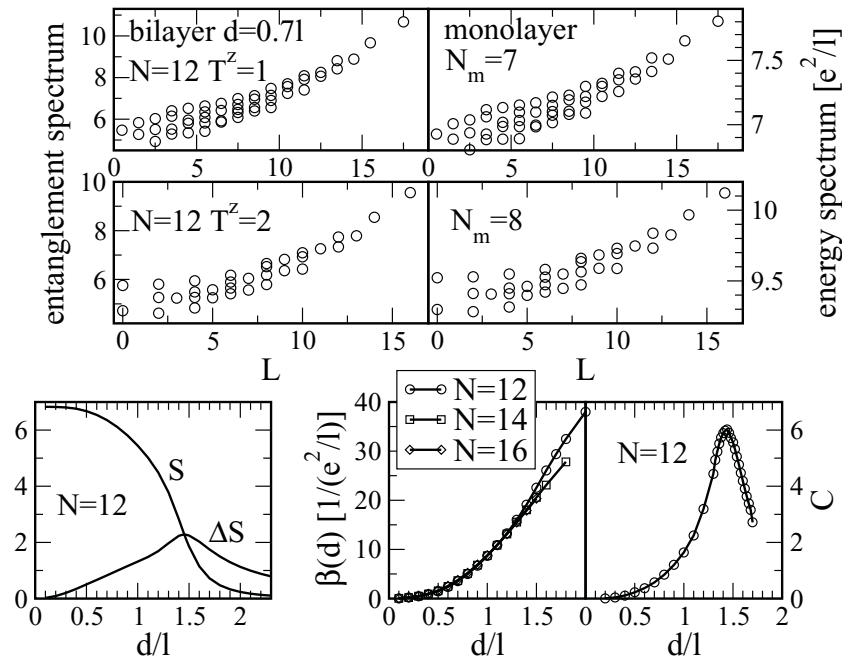

FIG. 4. Top panels: Entanglement spectra of $N=12$ electrons with $T^{z} \in\{1,2\}$ are compared with the corresponding monolayer spectra. Left bottom panel: Entanglement entropy $S$ along with its variance $\Delta S$ as a function of the layer separation $d$ for a balanced bilayer system of $N=12$ electrons. Right bottom panels: The "inverse temperature" $\beta$ and the "specific heat" $C$ as a function of layer separation.

level $\xi_{0}$, between a value of $S=\ln \left(\begin{array}{c}N \\ N / 2\end{array}\right)$ at $d=0$ and $S=$ $\ln \left(2 L_{0}+1\right)$ at large layer separation. In the left bottom panel of Fig. 4 we have plotted $S$ along with its variance

$$
\Delta S=\sqrt{\left\langle\left(-\ln \rho_{\text {red }}-S\right)^{2}\right\rangle}
$$

as a function of $d$ for a bilayer system of $N=12$ electrons. The entropy $S$ shows an inflection point, accompanied by a maximum of $\Delta S$, near $d=1.4 \ell$, which is close to the finitesize value of the phase boundary obtained earlier from an analysis of the pseudospin fluctuations. ${ }^{9,36}$

The observation that the entanglement spectrum of interlayer-coherent quantum Hall bilayers shows an intriguing similarity to the the energy spectrum of the corresponding monolayer extends also to unbalanced systems. This is illustrated in the top panels of Fig. 4 where the entanglement spectra of $N=12$ electrons with $T^{z} \in\{1,2\}$ obtained by tracing out the top layer are compared with the corresponding monolayer spectra. Entanglement spectra obtained from bilayer systems with negative $T^{z}$ (or, alternatively, by tracing out the bottom layer) are related to the previous ones by a particle-hole transformation are therefore identical, and the corresponding monolayer energy spectra just differ by an additive constant.

The investigations so far have concentrated on the case of vanishing single-particle tunneling between the layers. At finite tunneling amplitude, the particle number of each layer is no longer a good quantum number, and the monolayer Fock space consists of invariant subspaces of the reduced density matrix which are characterized both by total angular momentum and particle number. Therefore, for not too large tunneling, the entanglement spectra within each subspace of given particle number will be similar to those given in Figs 1, 2, and 3 up to an additive constant describing the spectral weight of the respective subspace.
Focusing again on the case of zero tunneling, our results suggest that the reduced density matrix fulfills, for not too large layer separation $d$, the following approximate relation:

$$
-\ln \rho_{\text {red }} \approx \beta \mathcal{H}_{m}+\ln Z,
$$

where $Z=\operatorname{tr} \exp \left(-\beta \mathcal{H}_{m}\right)$, and $\mathcal{H}_{m}$ describes the Coulomb repulsion in a half-filled monolayer with $N$ flux quanta and $N_{m}=N / 2$ electrons. The (inverse) "entanglement temperature" $\beta$ is a parameter depending on $d$, and the occurrence of the partition function $Z$ in Eq. (6) ensures the condition $\operatorname{tr} \rho_{\text {red }}=1$. Clearly, $d \rightarrow 0$ implies $\beta \rightarrow 0$ and $Z=\left(\begin{array}{c}N \\ N / 2\end{array}\right)$. The mid-bottom panel of Fig. 4 shows least-mean-square results for $\beta$ as a function of layer separation. As seen there, the data depend only very weakly on system size. Moreover, defining a "entanglement free energy" by $F=-T \ln Z, T=1 / \beta$, one derives from Eq. (6) the familiar thermodynamic relation

$$
F \approx E-T S
$$

with $E=\left\langle\mathcal{H}_{m}\right\rangle$. Thus, the entanglement entropy of the bilayer system turns out to have an immediate thermodynamic meaning for the monolayer system characterized by the parameter $T=1 / \beta$. These findings suggest to define, in full analogy with canonical thermodynamics, a "specific heat" via

$$
C=T \frac{d S}{d T}=-\beta \frac{d S}{d \beta} .
$$

In the mid-bottom panel of Fig. 4 we have plotted $C$ as a function of layer separation for a system of $N=12$ electrons. as seen, the specific heat has a pronounced peak near the phase boundary which should be seen as a strong hint for the underlying phase transition being first order. Finally, from Eq. (6) one also derives the result

$$
(\Delta S)^{2} \approx \beta^{2}\left(\left\langle\mathcal{H}_{m}^{2}\right\rangle-\left\langle\mathcal{H}_{m}\right\rangle^{2}\right)
$$

which provides a direct link between the variance of the entanglement entropy of the bilayer system and the energy variance of the monolayer. As seen in Fig. $4, \Delta S$ shows a maximum at the phase boundary which should be considered as another piece of evidence that, in the infinite-volume limit, the phase transition is of first order ${ }^{9}$ rather than continuous, ${ }^{11}$ i.e., the entropy changes discontinuously.

Very recently, Poilblanc has performed a study of entanglement spectra of Heisenberg spin ladders, where a similarly striking resemblance to the energy spectra of single spin chains was found. ${ }^{25}$ This observation could also be described in terms of an effective temperature. The results of both papers raise the question of a common underlying mechanism being at work, also extending previous studies on fractionally filled quantum Hall monolayers ${ }^{17-20}$ to bilayers at filling factor $v=1$ investigated here. Most interestingly, the latter direction of work makes immediate contact to the enigmatic system of a half-filled quantum Hall monolayer. However, here we should add the caveat that the monolayer systems that naturally arise in our present work contain $N$ electrons in $N_{\phi}=2 N-1$ flux quanta whereas in many previous numerical studies on (electron-spin polarized) monolayers at half-filling considered using spherical geometry other relations of the form $N_{\phi}=$ $2 N-M, M$ being a small integer, have been considered. ${ }^{37}$ To eliminate such a finite-size "shift problem" it would be 
desirable to repeat the calculations reported on here in the toroidal geometry.

We note that a different recipe of how to eliminate finite-size properties from entanglement spectra of quantum Hall monolayers in the spherical geometry was put forward very recently by Thomale et al. ${ }^{19}$ These authors propose to strip all normalization factors from single-particle states entering the Slater determinants of many-body wave functions. This procedure of achieving a "conformal limit" conserves azimuthal symmetry on the sphere (as appropriate for the situation of Ref. 19), but destroys full rotational covariance, which is desired in the present study on bilayer systems. Thus, the prescription proposed in Ref. 19 is unfortunately not applicable to our investigations here.

\section{CONCLUSIONS}

In summary, we have studied the entanglement spectra of bilayer quantum Hall systems at total filling factor $v=1$. Our investigations are based on exact numerical diagonalizations using the spherical geometry. In the interlayer-coherent phase at small layer separations, the entanglement spectra show a striking similarity to the energy spectra of the corresponding monolayer systems around half-filling. The transition to the incoherent phase can be followed in terms of lowlying entanglement levels, constituting a link between the entanglement spectrum and a quantum-phase transition. Clear signatures of the quantum-phase transition are also shown by the entanglement entropy along with its fluctuation. Moreover, the connection between those two types of spectra can be described in terms of an effective temperature which gives rise to relations for the entanglement entropy being fully analogous to canonical thermodynamics. In particular, the specific heat derived from this formalism provides a strong hint for the phase transition being of first order. ${ }^{9}$

\section{ACKNOWLEDGMENT}

This work was supported by Deutsche Forschungsgemeinschaft via SFB 631.
${ }^{1}$ S. Q. Murphy et al., Phys. Rev. Lett. 72, 728 (1994).

${ }^{2}$ I. B. Spielman, J. P. Eisenstein, L. N. Pfeiffer, and K. W. West, Phys. Rev. Lett. 84, 5808 (2000).

${ }^{3}$ M. Kellogg, I. B. Spielman, J. P. Eisenstein, L. N. Pfeiffer, and K. W. West, Phys. Rev. Lett. 88, 126804 (2003); M. Kellogg, J. P. Eisenstein, L. N. Pfeiffer, and K. W. West, ibid. 90, 246801 (2003); 93, 036801 (2004); A. R. Champagne, J. P. Eisenstein, L. N. Pfeiffer, and K. W. West, ibid. 100, 096801 (2008); A. D. K. Finck, J. P. Eisenstein, L. N. Pfeiffer, and K. W. West, ibid. 104, 016801 (2010).

${ }^{4}$ Y. Yoon, L. Tiemann, S. Schmult, W. Dietsche, K. von Klitzing, and W. Wegscheider, Phys. Rev. Lett. 104, 116802 (2010); R. D. Wiersma, J. G. S. Lok, S. Kraus, W. Dietsche, K. vonKlitzing, D. Schuh, M. Bichler, H. P. Tranitz, and W. Wegscheider, ibid. 93, 266805 (2004).

${ }^{5}$ S. Luin, V. Pellegrini, A. Pinczuk, B. S. Dennis, L. N. Pfeiffer, and K. W. West, Phys. Rev. Lett. 90, 236802 (2003); B. Karmakar, V. Pellegrini, A. Pinczuk, L. N. Pfeiffer, and K. W. West, ibid. 102, 036802 (2009); e-print arXiv:0907.4032.

${ }^{6}$ N. Kumada, K. Muraki, K. Hashimoto, and Y. Hirayama, Phys. Rev. Lett. 94, 096802 (2005); P. Giudici, K. Muraki, N. Kumada, Y. Hirayama, and T. Fujisawa, ibid. 100, 106803 (2008); P. Giudici, K. Muraki, N. Kumada, and T. Fujisawa, ibid. 104, 056802 (2010).

${ }^{7}$ K. Moon, H. Mori, K. Yang, S. M. Girvin, A. H. MacDonald, L. Zheng, D. Yoshioka, and S. C. Zhang, Phys. Rev. B 51, 5138 (1995); K. Yang, K. Moon, L. Belkhir, H. Mori, S. M. Girvin, A. H. MacDonald, L. Zheng, and D. Yoshioka, ibid. 54, 11644 (1996).

${ }^{8}$ S. He, S. Das Sarma, and X. C. Xie, Phys. Rev. B 47, 4394 (1993).

${ }^{9}$ J. Schliemann, S. M. Girvin, and A. H. MacDonald, Phys. Rev. Lett. 86, 1849 (2001).

${ }^{10}$ K. Nomura and D. Yoshioka, Phys. Rev. B 66, 153310 (2002); N. Shibata, Prog. Theor. Phys. Suppl. 176, 182 (2008).

${ }^{11}$ N. Shibata and D. Yoshioka, J. Phys. Soc. Jpn. 75, 043712 (2006).

${ }^{12}$ T. Nakajima, Phys. Rev. B 65, 233317 (2002).

${ }^{13}$ J. Schliemann, Phys. Rev. B 67, 035328 (2003).
${ }^{14}$ S. H. Simon, E. H. Rezayi, and M. V. Milovanovic, Phys. Rev. Lett. 91, 046803 (2003).

${ }^{15}$ G. Möller, S. H. Simon, and E. H. Rezayi, Phys. Rev. Lett. 101, 176803 (2008); Phys. Rev. B 79, 125106 (2009).

${ }^{16}$ L. Amico et al., Rev. Mod. Phys. 80, 517 (2008).

${ }^{17}$ H. Li and F. D. M. Haldane, Phys. Rev. Lett. 101, 010504 (2008).

${ }^{18}$ A. M. Läuchli, E. J. Bergholtz, J. Suorsa, and M. Haque, Phys. Rev. Lett. 104, 156404 (2010).

${ }^{19}$ R. Thomale, A. Sterdyniak, N. Regnault, and B. A. Bernevig, Phys. Rev. Lett. 104, 180502 (2010).

${ }^{20}$ A. Sterdyniak, N. Regnault, and B. A. Bernevig, e-print arXiv: 1006.5435.

${ }^{21}$ P. Calabrese and A. Lefevre, Phys. Rev. A 78, 032329 (2008).

${ }^{22}$ F. Pollmann and J. E. Moore, New J. Phys. 12, 025006 (2010).

${ }^{23}$ F. Pollmann, A. M. Turner, E. Berg, and M. Oshikawa, Phys. Rev. B 81, 064439 (2010).

${ }^{24}$ R. Thomale, D. P. Arovas, and B. A. Bernevig, Phys. Rev. Lett. 105, 116805 (2010).

${ }^{25}$ D. Poilblanc, Phys. Rev. Lett. 105, 077202 (2010).

${ }^{26}$ H. Yao and X.-L. Qi, Phys. Rev. Lett. 105, 080501 (2010).

${ }^{27}$ L. Fidkowski, Phys. Rev. Lett. 104, 130502 (2010).

${ }^{28}$ E. Prodan, T. L. Hughes, and B. A. Bernevig, Phys. Rev. Lett. 105, 115501 (2010).

${ }^{29}$ Z. Liu et al., e-print arXiv:1007.0840.

${ }^{30}$ F. D. M. Haldane, Phys. Rev. Lett. 51, 605 (1983).

${ }^{31}$ A. Burkov et al. Physica E 12, 28 (2002).

${ }^{32}$ B. I. Halperin, Helv. Phys. Acta 56, 75 (1983).

${ }^{33} \mathrm{We}$ do not show here complete entanglement spectra for layer separations larger than $d=1.3 \ell$ since in such situations, in particular at larger system sizes, the high-lying entanglement levels correspond to exceedingly small eigenvalues of the reduced-density matrix whose precise computation tends to overdemand the available numerical accuracy. The low-lying entanglement levels shown in Fig. 3 are not affected by this problem.

${ }^{34}$ See, e.g., L. D. Landau and E. M. Lifshitz, Quantum Mechanics (Butterworth Heinemann, Oxford, UK, 2005). 
${ }^{35} \mathrm{~A}$ similar scheme of adding angular momenta of single layers was also found for the electronic spin in quantum Hall bilayers at total filing factor $v=2$, J. Schliemann and A. H. MacDonald, Phys. Rev. Lett. 84, 4437 (2000).
${ }^{36} \mathrm{We}$ expect those slightly differing finite-size values for the phase boundary to converge in the thermodynamic limit.

${ }^{37}$ See, e.g., Ref. 15 and E. Rezayi and N. Read, Phys. Rev. Lett. 72, 900 (1994); R. H. Morf, ibid. 80, 1505 (1998). 